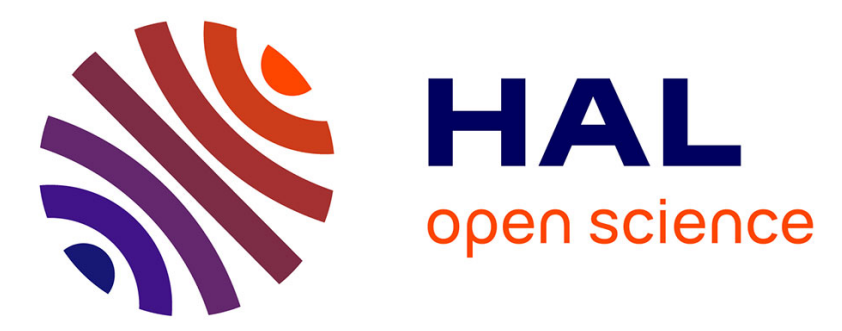

\title{
Identification of a new complex deleterious mutation in exon 18 of the gene in a hereditary male/female breast cancer family
}

Orland Diez, Sara Gutiérrez-Enríquez, Miriam Masas, Anna Tenés, Carme Yagüe, Angels Arcusa, Gemma Llort

\section{To cite this version:}

Orland Diez, Sara Gutiérrez-Enríquez, Miriam Masas, Anna Tenés, Carme Yagüe, et al.. Identification of a new complex deleterious mutation in exon 18 of the gene in a hereditary male/female breast cancer family. Breast Cancer Research and Treatment, 2010, 123 (2), pp.587-590. 10.1007/s10549-010-08302. hal-00517925

\section{HAL Id: hal-00517925 \\ https://hal.science/hal-00517925}

Submitted on 16 Sep 2010

HAL is a multi-disciplinary open access archive for the deposit and dissemination of scientific research documents, whether they are published or not. The documents may come from teaching and research institutions in France or abroad, or from public or private research centers.
L'archive ouverte pluridisciplinaire HAL, est destinée au dépôt et à la diffusion de documents scientifiques de niveau recherche, publiés ou non, émanant des établissements d'enseignement et de recherche français ou étrangers, des laboratoires publics ou privés. 
Identification of a new complex deleterious mutation in exon 18 of the $B R C A 2$ gene in a hereditary male/female breast cancer family

Orland Diez ${ }^{1,2}$, Sara Gutiérrez-Enríquez ${ }^{2,3}$, Miriam Masas ${ }^{1}$, Anna Tenés ${ }^{2}$, Carme Yagüe $^{4}$, Angels Arcusa ${ }^{4}$, Gemma Llort ${ }^{4}$.

${ }^{1}$ Oncogenetics Laboratory, University Hospital Vall d'Hebron, Barcelona. Spain.

${ }^{2}$ Vall d'Hebron Institute of Oncology (VHIO). Barcelona. Spain.

${ }^{3}$ Medical Oncology Program. Research Institute, Vall d'Hebron University Hospital. Barcelona. Spain.

${ }^{4}$ Genetic Counseling Unit. Consorci Sanitari de Terrassa. Vallès Oncologic Institute. Barcelona. Spain.

Corresponding author:

Orland Diez

Oncogenetics Laboratory

University Hospital Vall d'Hebron

08035 Barcelona

Spain.

Telf: 932746000 ext: 4826

Fax: 93-274 6837

Odiez@vhebron.net 


\begin{abstract}
We report a novel complex mutation that consists of a deletion of $12 \mathrm{bp}$ and an insertion of 2 bp (c.8402_8413del12ins2bp) in the exon 18 of the BRCA2 gene. This is a frameshift mutation that causes a disruption of the translational reading frame resulting in a stop codon downstream in the 2729 position of the BRCA2 protein. The mutation was present in a Spanish hereditary male/female breast cancer family.
\end{abstract}

\title{
Key words
}

$B R C A 2$, hereditary breast cancer

\section{Introduction}

Breast cancer is the most commonly occurring cancer among women. Many risk factors have been identified but a family history of breast cancer is one of the main risk factors for developing the disease.

It is currently estimated that about $5-10 \%$ of breast cancers are due to an inherited predisposition, and approximately $20-25 \%$ of this risk is explained by two high penetrance susceptibility genes: BRCA1 (17q21) (MIM\# 113705) [1] and BRCA2 (13q12-13) (MIM\# 600185) [2]. Both are tumor suppressor genes implicated mainly in DNA repair. Mutations in BRCA1 or BRCA2 account for a lifetime risk of approximately $40-65 \%$ for breast cancer and $11-40 \%$ for ovarian cancer [3]

Extensive analyses of the BRCA1 and $B R C A 2$ genes have resulted in the identification of a large number of different disease-causing germ-line mutations (listed online at the Breast Cancer Information Core) [4]. Most reported disease-associated alleles of $B R C A 1$ and $B R C A 2$ consist of small insertions or deletions that generate the occurrence of premature stop codons leading to truncation of BRCA1 or BRCA2 proteins. Less commonly, deletion/insertion (delins) complex mutations have also been described in both genes. Furthermore, a large number of missense, in-frame, and intron variants (especially those affecting splice sites positions) are routinely encountered in clinical and research laboratories, and cannot be readily distinguished as either diseaseassociated mutations or benign polymorphisms, and they are classified as variants of uncertain significance (BIC database).

We report a novel complex mutation that consists of a deletion of $12 \mathrm{bp}$ and an insertion of 2 bp (c.8402_8413del12ins2bp) in the exon 18 of the BRCA2 gene in three women belonging to a Spanish hereditary male/female breast cancer family.

\section{Patients}

The proband was diagnosed of breast cancer at 37 years of age (infiltrating ductal carcinoma, pT2 pN3a, GIII, ER-, PgR- HER2-), with non evidence of disease at 7 years of diagnosis. She has two sisters affected, one of them by breast cancer at 34 years (infiltrating ductal carcinoma, pT1cpN1, GIII, ER+, PgR+, HER2-). She is free of disease at 2 years from diagnosis. The other sister was diagnosed of synchronous bilateral breast cancer at 36 years (left breast: infiltrating lobular carcinoma, pT2 pN3a, GII, ER-, PgR+ HER2-, and right breast: infiltrating ductal carcinoma, pT1c pNx, GII, ER-, PgR+ HER2-) and two years after surgery she has been diagnosed of bone metastasis. Their father died at 52 years of metastatic breast cancer diagnosed at 46 years of age, and a paternal aunt was diagnosed with left breast cancer (pT2a pN0, GII, RE negative, RP positive) at 28 years and bilateral papillary serous ovarian carcinoma at 
age of 45 . The paternal grandmother presented breast cancer at the age of 70 . Figure 1 shows the pedigree of the affected family.

\section{Methods}

As part of genetic studies carried out on breast/ovarian Spanish families from the Genetic Counseling Unit in the Institut Oncologic del Vallès, germ-line BRCA1 and $B R C A 2$ mutations were screened in a proband of each family. Individuals underwent genetic counseling and signed an informed consent prior to testing. The molecular analyses were done in the Oncogenetics laboratory at the University Hospital Vall d'Hebron, Barcelona, Spain.

Genomic DNA was extracted from whole blood using the Puregene Genome DNA purification kit (Gentra System, Minneapolis, MN, USA). DNA was amplified by PCR using primers specific for the coding sequence and exon/intron boundaries. Analysis of the entire coding and flanking sequences was carried out by sequencing using BigDye Terminator V3.1 Cycle Sequencing Kit on a 3130xl Genetic Analyzer (Applied Biosystems, Foster City, CA, USA). Both forward and reverse strands were sequenced. The variant sequence described in our study is named according to the GenBank (BRCA2 NM_000059).

\section{Results}

A novel complex mutation was identified in the exon 18 of the BRCA2 gene: a deletion of $12 \mathrm{bp}$ and an insertion of $2 \mathrm{bp}$ (c.8402_8413delGGTATGCTGTTAinsTT) (figure 2) in the index case of a hereditary male/female breast cancer family. After identification of the alteration in the proband the analysis was then carried out on DNA samples obtained from two affected sisters. The same change was detected in the DNA of the two relatives. This alteration is not present in the BIC database nor has been documented in any published report to the best of our knowledge.

Furthermore, all mutation carriers analyzed in the family presented the variant c.296-7T >A in intron 2 of $B R C A 2$, that causes an alternative transcription with the skipping of exon 3 , that is present in normal population and is not considered as pathogenic $[5,6]$.

\section{Discussion}

Since the identification of BRCA1 and BRCA2, more than 3600 distinct genetic variants (including deleterious mutations, polymorphisms, and unclassified variants) have been reported for these genes (BIC database) [4] The great majority are small deletions or insertions that originate premature stop codons and lead to truncated proteins or mRNA degradation. A short number of delins frameshift alterations have also been described across the sequence of both genes (eighteen in BRCAl and fifteen in $B R C A 2)$.

As part of genetic studies carried out on breast/ovarian Spanish families we identified a new delins frameshift alteration in BRCA2 (c.8402_8413del12ins2bp) that causes a disruption of the translational reading frame resulting in a premature stop codon in the 2729 position. According to the HGV rules [7], it can be assumed that this change has occurred as the consequence of one mutational event, although the same alteration could have occurred as two sequence variants in the same allele: a c.8402_8410del9bp in frame deletion, and a frame shift c.8414delA mutation. The in- 
frame deletion of nine nucleotides would entail the absence of three amino acids (TrpThr-Ala) from codon 2725 to 2727 in the corresponding BRCA2 protein. This modification would have an unknown biological significance. The c.8414delA mutation would result in the appearance of the same stop codon in position 2729 , with a clear pathogenic role. Up to the date none of these two potential allelic variants (c.8402_8410del9bp and c.8414delA) have been described and the simplest HGV nomenclature must be opted.

However, many other presumably deleterious alterations have been reported in its vicinity, in exon 18. The BIC database reports 26 different pathogenic mutations and 43 changes with unknown significance, including two in frame deletions (p.K2750del and p.RLTVG2743del). This last mutation was predicted to result in the deletion of five amino acids (positions 2744-2748) in the carboxyterminal region of the protein, and was found to track exclusively in affected members of a breast/ovarian cancer family [8]. The region of BRCA2 harboring the mutation reveals high evolutionary conservation across species. This region corresponds to the 800-residue carboxylterminal domain of BRCA2, which is likely to play an important role in the tumor suppressor functions of the gene. It includes a helical domain (mostly $\alpha$-helices) followed by three structurally homologous domains that contain three oligonucleotide/oligosaccharide-binding (OB) folds (OB1, OB2, and OB3). The OB2 has a 130-amino acid insertion that adopts a tower-like structure protruding away from the OB fold [9]. The DSS1 protein, that may have a stabilizing function of BRCA2 within the cell, binds BRCA2 in an extended conformation interacting with the helical domain, $\mathrm{OB} 1$ and $\mathrm{OB} 2$ domains. Bearing in mind that it is the most conserved portion of $B R C A 2$ across different species and contains a high number of missense mutations found in breast cancer families, it seems reasonable to suggest the changes that occur in this region (even in frame or missense alterations) may be associated with a higher probability to an increase of risk of cancer in the carrier families.

The delins rearrangement here described presumably affects important BRCA2 protein functions since it causes the absence of the C-terminal conserved region in the protein, that mediate binding to RAD51 (directly involved in homologous recombination), and some other proteins like DSS1 [10,11]. Alternatively, the mutation could originate the degradation of the transcripts by the mRNA nonsense mediated decay mechanism. Consequently, the mutation can be classified as clearly pathogenic and associated to a high risk of female and male breast cancer and ovarian cancer in agreement with the phenotype of the studied family.

In this pedigree, the BRCA2 (c.8402_8413del12ins2bp) mutation cosegregates with breast cancer in the three sisters, all of them affected before 40 years. The age at diagnosis of the breast cancer of the father (46 years) is younger that the mean age of onset of male breast cancer reported in mutation positive families (62.1 years) [12].

It has not been possible to test the father and the paternal aunt (affected by two primary tumors: very early breast cancer at 28 years, and bilateral ovarian cancer diagnosed at 45 years), that probably carry the familiar mutation. The risk of ovarian cancer in $B R C A 2$ carriers is very low at 50 years of age, but increases sharply in the 5059 years age group, perhaps declining somewhat thereafter [3]. Together, these findings strongly suggest that the BRCA2 c.8402_8413del12ins2bp mutation is of high penetrance. 


\section{Acknowledgements}

This work was in part funded by a grant (2008) from Fundación de Investigación Médica Mutua Madrileña.

\section{References}

1-Miki Y, Swensen J, Shattuck-Eidens D et al (1994) A strong candidate for the breast and ovarian cancer susceptibility gene BRCA1. Science 266:66-71.

2-Wooster R, Bignell G, Lancaster J et al (1995) Identification of the breast cancer susceptibility gene BRCA2. Nature 378:789-792.

3-Antoniou A, Pharoah PDP, Narod S et al (2003) Average risks of breast and ovarian cancer associated with BRCA1 or BRCA2 mutations detected in case series unselected for family history: A combined analysis of 22 studies. Am J Hum Genet 72:1117-1130.

4-BIC: http://research.nhgri.nih.gov/bic.

5-Koul A, Nilbert M, Borg A (1999) A somatic BRCA2 mutation in RER+ endometrial carcinomas that specifically deletes the amino-terminal transactivation domain. Genes Chromosomes Cancer 24:207-212

6-Santarosa M, Viel A, Boiocchi M (1999) Splice variant lacking the transactivation domain of the BRCA2 gene and mutations in the splice acceptor site of intron 2. Genes Chromosomes Cancer 26:381-382.

7-Human Genome Variation Society: http://www.hgvs.org/rec.html

8-Martinez SL, Herzog J, Weitzel JN (2004) Loss of five amino acids in BRCA2 is associated with ovarian cancer. $\mathrm{J}$ Med Genet 41:e18.

9-Yang H, Jeffrey PD, Miller J, Kinnucan E, Sun Y, Thoma NH, Zheng N, Chen PL, Lee WH, Pavletich NP (2002) BRCA2 function in DNA binding and recombination from a BRCA2-DSS1-ssDNA structure. Science 297:1837-1848.

10- Pellegrini L, Yu DS, Lo T, Anand S, Lee M, Blundell TL, Venkitaraman AR (2002) Insights into DNA recombination from the structure of a RAD51-BRCA2 complex. Nature 420:287-293.

11-Marston NJ, Richards WJ, Hughes D, Bertwistle D, Marshall CJ, Ashworth A (1999) Interaction between the Product of the Breast Cancer Susceptibility Gene BRCA2 and DSS1, a Protein Functionally Conserved from Yeast to Mammals. Mol Cell Biol 7:4633-4642.

12-Evans DGR, Bulman M, Young K, Howard E, bayliss S, Wallace A, Lalloo F (2008) BRCA1/2 mutation analysis in male breast cancer families from North West England. Fam Cancer 7:113-117.

Figure 1: Family pedigree: $\mathrm{BC}$ breast cancer. Between parenthesis: age at diagnosis. +: carrier of the BRCA2 c.8402_8413del12ins2bp mutation.

Figure 2: Partial electropherogram of the BRCA2 exon 18 PCR product showing the wild type and the mutated alleles, and scheme of the changes in the nucleotide sequence. 
Fig. 1

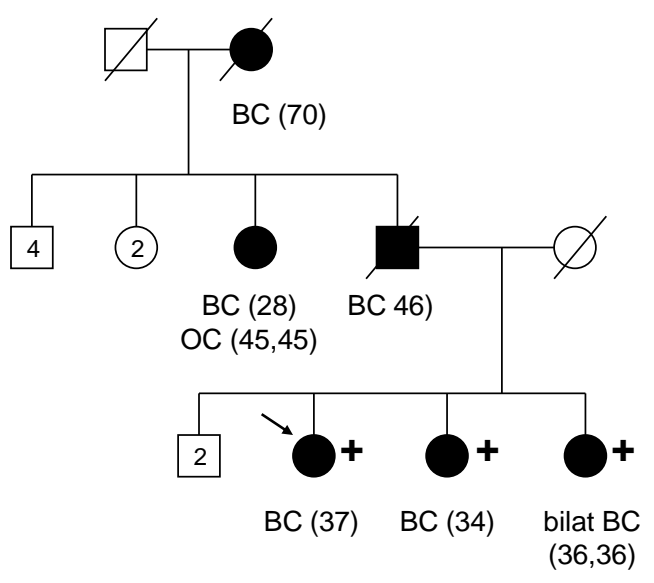

Fig. 2

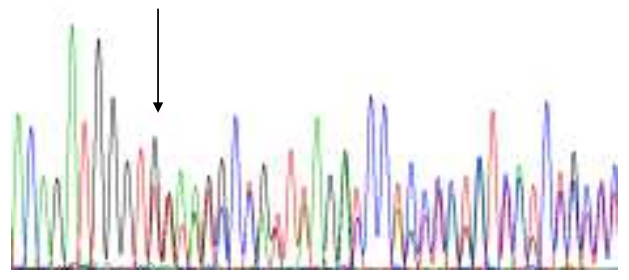

del12bp

---GGG TGG TAT GCT GTT AAG GCC CAG TTA GAT---

---GGG TITAGG CCC AGT TAG

ins $2 b p$ 